\title{
REDUCTION OF INSAR DEM TROPOSPHERIC NOISE WITH GPS OBSERVATIONS
}

\author{
A. Sharifi ${ }^{1 *}$, M. Hosseingholizadeh ${ }^{2}$ \\ ${ }^{1}$ Department of Surveying engineering, Faculty of civil engineering, Shahid Rajaee Teachers Training University, Tehran, \\ a_sharifi@sru.ac.ir \\ ${ }^{2}$ Department of Surveying engineering, Faculty of civil engineering, Shahid Rajaee Teachers Training University, Tehran, \\ m.hgzadeh@gmail.com
}

KEY WORDS: Interferometric SAR, Digital elevation model, Tropospheric noise, GPS, Accuracy assessment

\begin{abstract}
:
Satellite Interferometric Synthetic Aperture Radar (InSAR) Signals are often intensively contaminated by atmospheric delays. The atmospheric conditions especially its water vapor content significantly varies in space and with time. Therefore, it is essential to characterize the atmospheric variations in order to mitigate these effects by appropriate means. In the topographic case, for high-resolution DEM generation, the errors may be decreased by choosing interferometric pairs with relatively long baselines, as the error magnitude is inversely dependent on the perpendicular component of the interferometer baseline. For InSAR DEM quality estimation against tropospheric corrected one, we use the GPS measurements to create the reference DEM in the region. The preliminary results show that the corrected DEM by the phase gradient method has the highest correlation with the groundbased observations and the InSAR DEM which is corrected by phase gradient stacking method for water vapor effect in the troposphere has the best accuracy, ranging from $0.07 \sim 5.81 \mathrm{~m}$, while the InSAR original DEM has an accuracy of $0.29 \sim 14.62 \mathrm{~m}$.
\end{abstract}

\section{INTRODUCTION}

Structural and erosion related processes interaction through geological periods is the primary cause for a planet surface formation. The surface morphology estimation is the first step in understanding these processes. The erosion amount often depends on local slop angles, and the river drainage network, which is controlled by topography. Thus, the quantization of surface form is one of the necessities. Topography is essential in many anthropogenic activities, from transportation infrastructures to flood and landslide mitigation.

Topography maps have a long history that starts with a qualitative display of hills, valleys, and mountains, and then converts to quantitative topography contour maps. At the beginning of the twentieth century, the advent of stereo aerial photography makes it possible to measure the photogrammetric heights, and a large number of areas are mapped in various scales by these tools. The stereo images or photos achieved by satellites are then used for topographic maps production, and a large extent of earth surface is mapped in the minimum scale of 1:1000000. The main problems in optical imaging of some regions are the cloud coverage, and lack of suitable contrast among features in glacier districts, that prohibit the appropriate imaging.

However, the first Digital Elevation Model (DEM) is formed by digitizing the topographic map contours, and the interpolation of elevations for construction of a conventional grid. This method is also used for regions with high-quality contour maps yet. A high-resolution DEM facilitates the information extraction in different applications. The new technology of Radio Detection and Ranging (RADAR) Interferometry can be used for high-quality DEMs. Applications such as flood estimation, landslide detection, surface morphology mapping, and underground mining subsidence monitoring are some of the certain DEMs advantages.

\section{DATA SET}

Our proposed test site is the Bam city, an arid volcanic region in Iran, Southwestern Asia. The site includes both relatively flat, homogeneous regions as well as mountainous discontinuities. For stereo analysis and DEM construction, our interferometric data consists of Envisat interferometric pairs with a temporal baseline of 35 days. The corresponding height ambiguities are $17 \mathrm{~m}$. All images were resampled to a ground resolution of approximately $30 \mathrm{~m}$ in both dimensions. Various Multi baseline interferograms are produced for proposed atmospheric noise reduction technique. For evaluation, we used a reference DEM that was derived from the GPS real-time point measurements in the region. The DEM has an estimated accuracy of 20-25 cm. It should be noted that our error analysis concentrates on obvious interferometric errors on the order of one to two-phase cycles for atmospheric induced noise.

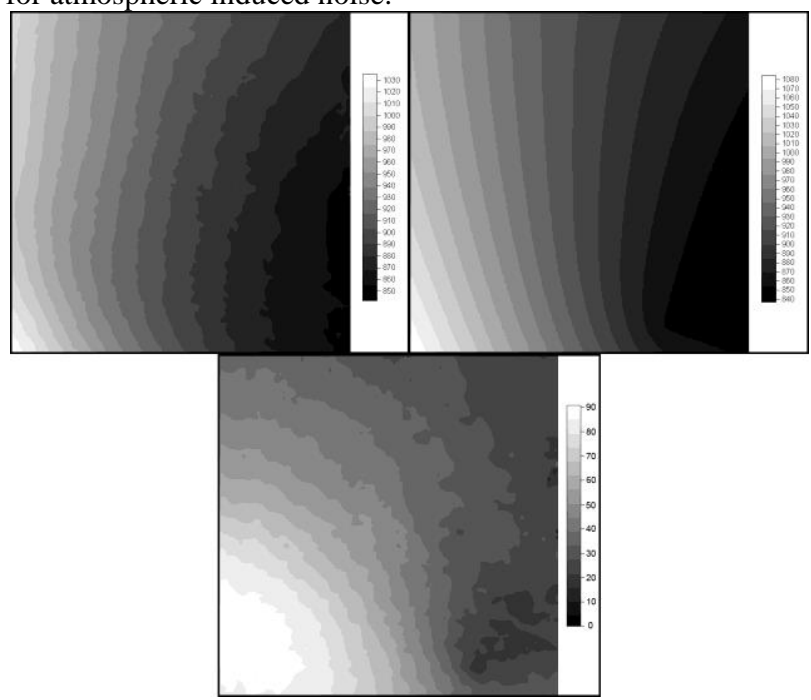

Figure 1. a. DEM before the time of SAR acquisition. b. DEM

\footnotetext{
* Corresponding author
} 
after the time of SAR acquisition. c. Elevation difference surface generation from GPS geodetic points.

\section{METHODOLOGY}

\subsection{Synthetic Aperture RADAR Interferometry}

Synthetic Aperture RADAR (SAR) is an active imaging system, which implies the microwave portion of the electromagnetic spectrum with 0.3 to $300 \mathrm{GHz}$ frequency range $(1 \mathrm{~m}$ to $1 \mathrm{~mm}$ wavelength). Each SAR image includes the amplitude (brightness) and phase information of backscattered signals. Interferometric SAR requires two SAR images that were taken from the same area. These two images can be achieved by two separate antennas mounted on airborne platforms and spaceborne systems, or by one antenna, which revisits the same region twice. These two images are then co-registered with each other in order to calculate the phase difference of corresponding pixels in them. Interferograms are often displayed overlaying the brightness rendered in grayscale, and phase as color, where each cycle of color represents a phase change of 2 radians. This phase difference or interferogram can be used for DEM extraction of the proposed area.

Differences in range distance can be determined by multiplying the relative phase differences in units of cycles by the wavelength of the radar signal. To assume the image point's position, the orbit information, information about the baseline vector separating the two imaging antenna positions, and the interferometric range are required. Due to consistency with surface deformation measurements, the repeat-track or dual pass configuration is described. Our goal is to determine each image point elevation $\mathrm{h}$ accurately by two SAR images. The radar carrier wavelength is defined from radar system design. The flight height $\mathrm{H}$ and orbit separation vector can be determined from accurate satellite orbits. The initial satellite-to-image point range distance along the look vector is estimated from the radar signal delay and the relative difference among two phase measurements in the interferogram.

\subsection{InSAR DEM Production}

Digital Elevation Model is usually a gridded representation of the region's topography. InSAR methods for DEM generation are different according to the geometry of the transmitting and receiving antennas. Systems designed for topographic applications, usually apply two antennas configuration on the same platform (Graham, 1974; Zebker \& Goldstein, 1986). One antenna transmits the microwave signal and receives the backscattered energy by the same antenna and another one which is located at a fixed distance. Most spaceborne systems are based on single-antenna satellites, in which the spacecraft must firmly repeat the initial orbit to produce the second image for precious interferometry. According to Rosen et al., 2000 the interferometric phase is

$$
\phi=4 \pi \rho_{1} / \lambda\left[\left(1-2\left(\vec{B} \cdot \hat{l}_{1}\right) / \rho_{1}+\left(B / \rho_{1}\right)^{2}\right)^{2}-1\right](1)
$$

Where $\hat{l}_{1}$ is the unit vector in the range direction and $\vec{B}$ is the baseline separation vector of length $B$. If the baseline length is $B<<\rho_{1,2}$, we can apply a parallel ray approximation (Zebker\& Goldstein 1986) and simplify equation (1) to:

$$
\phi \cong-\frac{4 \pi}{\lambda} \cdot\left(\vec{B} \cdot \hat{l}_{1}\right)=-\frac{4 \pi}{\lambda} \cdot B \cdot \sin (\theta-\alpha)(2)
$$

That is, the interferometric phase approximately depends on the projection of the antenna baseline vector on the look direction (Zebker \& Goldstein 1986). The interferometry technique measures the coherent nature of SAR imaging for stereo parallaxes in millimeter to centimeter scale detection from phase differences. According to InSAR imaging scheme in figure1, the phase difference (Interferometric phase) is as follows:

$$
\Delta \phi=\phi_{2}-\phi_{1}=\phi_{\text {prop }, 2}-\phi_{\text {prop }, 1}=\frac{4 \pi}{\lambda}\left(R_{2}-R_{1}\right)(3)
$$

Where the scattering phase is constant ${ }^{\phi}$ pscatt1 $=\phi_{\text {pscatt2 }}$. It is evident that range parallax $\Delta R=R_{2}-R_{1}$ is a measure for look angle $\theta$, and it depends on terrain height $h$. Due to sensitivity relation of height to phase in repeat-pass across-track SAR interferometer,

$$
\frac{\partial \Delta \phi}{\partial h} \cong \frac{4 \pi B_{\perp}}{\lambda R \sin \theta}(4)
$$

It would be desirable to operate at a larger baseline. In other words, with baseline increase in two complex SAR images, the interferogram tends to decorrelation state, and needs for socalled spectral shift filtering (Gatelli et al.1994). When the spectral shift exceeds the SAR system bandwidth $\mathrm{W}$, the interferogram is completely decorrelated and useless for ground surface reconstruction. The maximum acceptable baseline, called critical baseline, presents as

$$
B_{\perp \text { crit }}=\frac{\lambda W R \tan (\theta-\alpha)}{c}(5)
$$

In which $\alpha$ is the local terrain slope component in range direction, and $C^{C}$ is the light velocity (Bamler, 1997). For ENVISAT, the critical baseline is about $1100 \mathrm{~m}$. The iso-phase lines, defined as fringes, resemble the iso-height lines on earth surface. However, geolocation of each interferogram pixel for DEM generation should not be applied before the 2-D phase unwrapping stage that removes the $2-\pi$ phase ambiguity. It should be noted that the phase information in every interferogram pixel, is available even in completely homogeneous regions that stereo methods based on contrast are not sufficient. With phase measurement, the known radar wavelength, and the geometry shown in figure1, we can determine $\theta=\sin ^{-1}(-\lambda \phi / 4 \pi B)+\alpha$, and the surface elevation is then $h=H-\rho_{1} \cos \theta$ (Burgmann et al.2000).

\subsection{InSAR DEM Accuracy}

The accuracy of interferometric DEM is determined by three main factors including pixel phase measurement accuracy (phase noise), imaging geometry accuracy (orbit errors), and consistency of wave propagation conditions (atmospheric artifacts) (Bamler, 1997).

\subsubsection{Phase Noise}

Interferometric phase noise $\boldsymbol{\sigma}_{\Delta \phi}$ induced a height error as follows:

$$
\sigma_{h} \cong \frac{\partial h}{\partial \Delta \emptyset} \sigma_{\Delta \emptyset} \cong \frac{\lambda R \sin \theta}{4 \pi B_{\perp}} \sigma_{\Delta \emptyset}(6)
$$

This kind of error varies relatively rapidly from pixel to pixel. 
The leading causes of phase noise are system noise like thermal receiver noise and quantization noise. These elements are the primary noise sources in single-pass interferometry (Burgmann, 2000). Temporal scene decorrelation in repeat-pass interferometry is another source for phase noise. In this case, the sub-resolution properties of the imaged scatterer may change between surveys. If the sub-pixel scattered' movement in a resolution cell is in the order of wavelength, the scattering phase components $\Phi_{\text {pscatt, 1,2 }}$ no longer cancel, and the two SAR images decorrelate.

Any phase noise source can be determined by the amount of decorrelation it introduces. The conventional measure for interferogram quality is coherence $\gamma$, defined as the mutual correlation coefficient between the two images (Just and Bamler, 1994), (Wegmuller et al.1995). Its greatness is bounded between 0(i.e., total decorrelation, no phase information) and 1(no phase noise). This noise can be reduced by spatially lowpass filtering the interferogram at the expense of horizontal resolution. Repeat-pass interferometers suffer from lack of coherence due to temporal decorrelation; coherence may take any values between 0 for water areas and approximately 0.9 for dry soil and rock.

\subsubsection{Orbit Errors}

Insufficient accuracy in sensor position levitates large-scale terrain reconstruction errors. The orbit error component that rotates the interferometer baseline effectively could also rotate the DEM. Therefore, orbit errors can cause horizontal and vertical shifts of the entire DEM as well as to ramp-like height distortion (Bamler, 1997). These distortions are more noticeable with increasing swath width. The conventional approach for this type of noise is the application of flat surfaces with known elevation, ground control points, or seashores as references to improve the baseline and orbit parameters. The inaccurate orbit and attitude problem are not specific to InSAR; it is preferably a geometric problem that also happens in optical imaging. Today spaceborne SAR orbital errors are about tens of centimeters, that are high for automatic DEM generation and ground control points are still required.

\subsubsection{Atmospheric Artifacts}

Due to the availability of interferometric SAR data and improvements of processing systems, propagation effects on the interferometric phase can be studied extensively. Repeat-pass interferometry is based on the constancy of the radar wave propagation conditions for two image acquisitions. Currently, some phenomena are introduced for phase delay errors including tropospheric water vapor, and ionospheric effect (Dupont, 1999).

Tropospheric water vapor may cause phase shifts in Envisat interferograms. In this case, cloud-like or ripple-like phase structures are found in a large number of data sets. The phase error is often smaller than 0.5 fringe cycles. This kind of error can be averaged because several interferograms of the same region are required for DEM generation.

In many SAR data, localized phase errors due to convective cells of water vapor have been observed. Their extent is minor than 30 kilometers. The phase error can be 1 to 3 fringes. Their particular structure makes it possible to identify them quickly and remove these areas from the interferogram (Zebker, 1996). The ionospheric effect on interferogram is not determined correctly. These waves are often appeared in arctic regions and propagate to lower latitudes.

\section{EXPERIMENTAL PROCEDURE}

\subsection{Interferometric DEM Generation}

The InSAR DEMs used for evaluation in this paper, derived from multi-baseline Envisat acquisitions. Each ASAR image pair has a short temporal separation, which reduces the local noise, vegetation growth, and surface displacement errors. The details of ASAR images and resulted interferograms are presented in Table 1.

For InSAR processing, three pairs of ASAR images (Table. I), taken at different baselines, are first co-registered to an accuracy of about $1 / 10$ of the pixel and then multiplied to form an interferogram. The interferometric phase is ambiguous concerning integer multiples of 2 and is usually displayed in a color-wheel fashion. The fringes resemble iso-height lines of the terrain. In this stage, the DEM generation can be carried out after the phase unwrapping to remove the 2-phase ambiguity. Atmospheric artifacts accompany these interferometric DEMs. The main atmospheric component responsible for these DEMs quality mitigation is water vapor.

Table 1. ASAR images and resulted interferograms

\begin{tabular}{|c|c|c|c|c|}
\hline Mission & Date(day/month/year) & orbit & track & Baseline(m) \\
\hline Envisat & $6 / 11 / 2003$ & 6687 & 120 & 0 \\
\hline ERS-2 & $6 / 12 / 2003$ & 2761 & 68 & 50 \\
\hline Envisat & $12 / 3 / 2003$ & 9192 & 120 & 40 \\
\hline ERS-2 & $12 / 4 / 2003$ & 2820 & 68 & 140 \\
\hline Envisat & $1 / 7 / 2004$ & 9693 & 120 & 0 \\
\hline ERS-2 & $1 / 8 / 2004$ & 2934 & 68 & 150 \\
\hline
\end{tabular}

\subsection{Tropospheric Artifacts Reduction}

Figure2 presents the three interferograms used for topographic mapping. The tropospheric artifacts can be determined in multi-baseline conditions because the atmosphere is correlated during the time. As these phase errors can be 1 to 3 fringes, and their extent is minor than 30 kilometers, we stacked the wrapped phases of two and three fringes respectively. The stacking method on water vapor induced phases through the phase gradient method provides a good result of merged and wrapped phases. Nevertheless, some problems caused by high frequency are still available in the phase gradient stacking results. For solving these problems, we applied the phase gradient using real phase instead of complex data to stack differential interferograms.

Interferograms stacking applies to increase fringe clarity and to decrease decorrelation error; however, phase filtering is needed for reduction of noise in case of low coherence or low signal to noise ratio. The stacked interferogram after filtering is shown in figure 3 has more evident fringe pattern than figure2.

\subsection{Assessment of DEMs against GPS data}

Global Positioning System is an instrument that can provide the instantaneous point coordinates with centimeter-level accuracy. Global positioning applies static GPS receiver as a reference station located at a known point. The other receiver is used as the rover who can move and survey any desired point. Both 
receivers make observations of GPS signals at the same time. The radio data link between two receivers makes it possible to send the data from reference to the rover, where the coordinate calculation is carried out. Despite InSAR for spatial measurement of surface height changes, GPS calculates this phenomenon continuously in time.

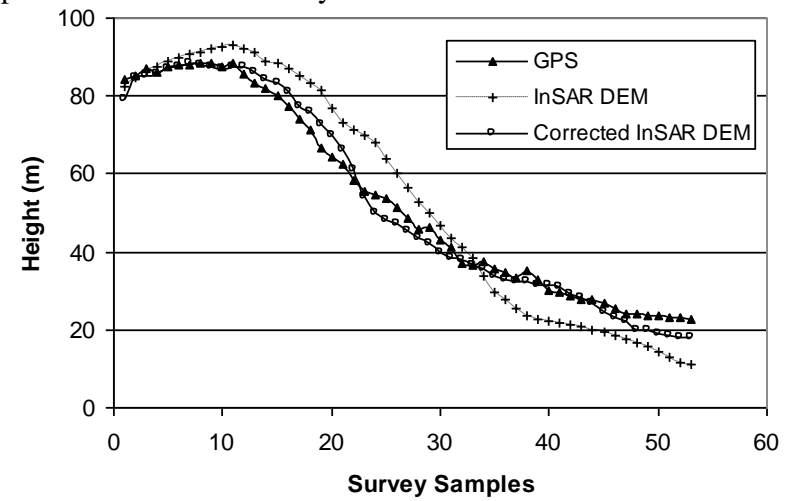

Figure 2. Three cross profiles along the maximum height difference. The graph shows the height difference (vertical axis) against the distance intervals (horizontal axis).

For InSAR DEMs assessment, the GPS point measurements of region's topography prior and post SAR data acquisitions were used. The samples with 3-dimensional accuracy better than 5 centimeters are only used for this procedure in Surfer v.8 software. The two GPS derived surfaces are then subtracted for elevation difference surface generation figure4. This surface called GPS DEM is used for validation of InSAR DEM modification algorithm.

In order to evaluate the three resulted DEMs, cross profiles in maximum height difference direction are derived initially. These three profiles are then presented in a diagram showing the height change versus distance in profile direction figure 2 . As it is presented, InSAR DEM which is corrected by phase gradient algorithm before phase unwrapping for tropospheric artifacts especially water vapor has better accuracy and higher quality than original InSAR DEM.

\section{CONCLUSION}

The accuracy of two DEMs derived using Interferometric Synthetic Aperture RADAR, tropospheric noise correct InSAR DEM, has been examined by comparison with GPS field survey results. Our results show that InSAR DEM which is corrected by phase gradient stacking method for water vapor effect in the troposphere has the best accuracy, ranging from $0.07 \sim 5.81 \mathrm{~m}$, while the InSAR original DEM has an accuracy of 0.29 $\sim 14.62 \mathrm{~m}$

\section{REFERENCES}

Bamler, R., 1997, Digital terrain models from radar interferometry, Photogrammetric Week, 97, 93-105.

Bergmann, R., Rosen, P.A. and Fielding, E.J., 2000, Synthetic aperture radar Interferometry to measure Earth's surface topography and its deformation. Annual Reviews Earth planet science, 28,169-209.
Colesanti, C., Ferretti, A., Novali, F., Prati, C., Rocca, F., 2003, SAR monitoring of progressive and seasonal ground deformation using the permanent scatterers technique, GeoRS(41), No. 7, pp. 1685-1701.

Dupont E., L. Menut, B. Carissimo, J. Pelon, and P. Flamant, 1999, Comparison between the atmospheric boundary layer in Paris and its rural suburbs during the ECLAP experiment. Atmos. Environ., 33, 979-994.

Gatelli, F., Monti-Guarnieri, A., Parizzi, F., Pasquali, P., Prati, C., and Rocca, F., 1994, The wavenumber shift in SAR interferometry. IEEE Transactions on Geoscience and Remote Sensing, 32(4):855-865.

Graham, L.C., 1974. Synthetic interferometer radar for topographic mapping, Proc. IEEE, 62, 763-768.

Just, D., and Bamler, R., 1994, Phase statistics of interferograms with applications to synthetic aperture radar, Appl. Opt. 33, 4361-4368.

Hansen, R. C., 1998, Phased array antennas. John Wiley \& Sons, Inc., New York.

Hong, S.H., Kim, S.W. and Won, J.S., 2003, Subsidence at Duk-Po area revealed by DInSAR and interferogram stacking. Department of Earth system sciences, Yonsei University, Seoul, Korea.

Massonnet, D., Briole, P. and Arnaud, A., 1995, Deflation of Mount Etna Monitored by Spaceborne Radar Interferometry, Nature, 375 (1995) 567-570.

Rosen, P.A., Hensley, S., Joughin, I.R., Li, F.K., Madsen, S.N., Rodriguez, E. and Goldstein R.M., 2000, Synthetic aperture radar interferometry. Proceeding of the IEEE, 88(3), 333-382.

Sandwell, D. T. and E. J. Price, 1998, Phase gradient approach to stacking interferograms, J. Geophys. Res., 103, 3018330204.

Tarayre Hélène, 1996, Extraction de modèles numériques de terrain par interférométrie radar satellitaire, algorithmie et artéfacts atmosphériques. PhD Thesis, MCS 1996.

Zebker, H.A., and Goldstein, R.M., 1986, Topographic mapping from interferometric SAR observations, J. Geophys. Res., 91 (B5), 4993-4999.

Zebker, H. and Rosen, P.A. 1996, Atmospheric artifacts in interferometric SAR surface deformation and topographic maps. Department of Electrical Engineering and Geophysics, University of Stanford, Stanford, CA.

Zebker, H. and Warner, C., Rosen, P.A. and Hensley, S. 1994 Accuracy of topographic maps derived from ERS-1 interferometric radar. IEEE Transactions on Geoscience and remote sensing, 32,823-836.

Wegmueller, S., Amberger, G. and J.-P. Vernet, 1995, La formation de Montfleury près de Genève : étude palynologique et sédimentologique d'un séquence du Pléistocène moyen. Ecologae geologicae Helvetiae 88/3, 595-614. 